\title{
The Paradigm of Universal Access to HIV-Treatment and Human Rights Violation: How Do We Treat HIV-Positive People Who Use Drugs?
}

\author{
Monica Malta $\cdot$ Michelle Ralil da Costa • \\ Francisco Inácio Bastos
}

Published online: 27 December 2013

(C) Springer Science+Business Media New York 2013

\begin{abstract}
HIV-positive people who use drugs (PWUDs) are particularly vulnerable for suboptimal access to highly active antiretroviral therapy (HAART). We conducted a systematic review to identify factors associated with suboptimal HAART access among this population. Studies evaluating HAART access among active PWUDs as a primary outcome, presenting multivariate analysis and conducted after January 1997 were included. Of 122 studies matching the search criteria, only $14(11.4 \%)$ met the inclusion criteria. All selected studies were prospective cohorts and included young adults, 13 were conducted in North America or western Europe and one in Ukraine. Selected studies measured HAART access using different strategies, however, all identified PWUDs as less likely to receive HAART, when compared to those who never used drugs or former PWUDs. Additional factors associated with suboptimal HAART access include: recent incarceration, lack of health insurance, unstable housing, depression, nonwhite ethnicity, female PWUDs, and health professionals stigma/prejudice. Factors associated with higher rates of HIV-treatment access included: alcohol and/or drug addiction treatment (especially methadone maintenance therapy), regular source of primary care, treatment and care from the same
\end{abstract}

\author{
M. Malta $(\bowtie)$ \\ Social Science Department, ENSP/FIOCRUZ, Rio de Janeiro, Brazil \\ e-mail: malta@ensp.fiocruz.br \\ M. Malta \\ e-mail: mmalta@jhsph.edu \\ M. Ralil da Costa \\ Belo Horizonte Observatory for Urban Health, Federal University of \\ Minas Gerais, Belo Horizonte, Brazil \\ e-mail: mralil@hotmail.com \\ F. I. Bastos \\ Health Information Department, ICICT/FIOCRUZ, Rio de Janeiro, \\ Brazil \\ e-mail: francisco.inacio.bastos@hotmail.com
}

provider (most of the time) and larger physician experience in HIV-management. PWUDs face a synergy of social and structural factors that influence their suboptimal access to HAART, struggling with poor living conditions, inadequate access to specialized care and stigma/discrimination from health professionals. Renewed strategies and effective interventions should be developed and scaled-up, in order to assure equitable HAART access, decrease morbidity and mortality among PWUDs.

Keywords HIV-treatment · HIV-treatment access $\cdot$ People who use drugs (PWUDs) · PWUDs · HIV-positive people · Highly active antiretroviral therapy (HAART) · HAART . Science of prevention · Human rights · People living with HIV (PLWHA) · Treatment · HIV · HIV-positive drug users . Drug use

\section{Introduction}

Improvement in quality of life of people living with HIV (PLWHA) under highly active antiretroviral therapy (HAART) has been comprehensively reviewed in recent years [1-3]. Findings from the HIV Prevention Trials Network 052 (HPTN 052) and subsequent studies also showed that early treatment of PLWHA not only improve the patients' health and their overall quality of life, but also dramatically cuts the rate of new infections $[4,5]$. Studies of communities with high concentrations of people who use drugs (PWUDs) and men who have sex with men (MSM) have also shown that as HAART use increased within the community, the community's viral load declined, as did rates of new HIV diagnoses [6, 7]. However ARV prophylaxis for PWUDs remains tentative and new protocols and/or additional analyses of existing protocols (e.g. The Bangkok Tenofovir Study) are sorely needed [8]. 
Following the same patterns identified among PLWHA, HIV-positive drug users who have access to timely treatment and achieve adequate adherence levels reach similar levels of viral suppression as the ones identified among other populations $[6,9,10]$. Resistance to one or more than one major class of antiretrovirals constitutes a major concern, worldwide, but no significant differences have been made evident between PLWHA who inject and do not inject drugs [11]. Similar rates of HIV-1 RNA suppression and rebound after HAART initiation have also been identified among HIV-positive drug users and patients who do not inject drugs [12]. However, in spite of growing evidence supporting the efficacy of early HAART initiation, drug users are disproportionately less likely to receive HAART than other patients, and levels of HAART access for active drug users are likely to be even lower, worldwide [13, 14].

Donoghoe and colleagues [15] evaluated HAART access among people who inject drugs (PWID) from 52 countries in the WHO European region, identifying a great inequity in drug users' access to HAART. Among 19 countries that provided data from 2002 to 2004, the authors identified a stable number of new infections among PWID: $45 \%$ in 2002 and $42 \%$ in 2004. Notwithstanding, the inequities in HAART access were striking: in 2002 only $5 \%$ of HAART recipients were PWID, 'increasing' to $6 \%$ in 2004. Grigoryan et al. [16] evaluated US national HIV surveillance data from 1996-2004. The study identified 27,572 PWID (42.2\%) who were diagnosed in a late stage. The three years survival rate after HIV diagnosis was lower for both male and female injection drug users, when compared to both men who have sex with men (MSM) and heterosexuals (male/female). The same study documented PWID also had a high risk for progression from HIV to AIDS.

Drug addiction is a chronic and relapsing condition, and a large percent of those patients usually experience periods of abstinence and relapses across their lifespan. As discussed in the seminal paper by McLellan [17], the course of this complex, multidimensional chronic disease does not markedly differ from other major chronic conditions, but such conceptual advances had not been translated into concrete improvements in drug abuse management [18]. Drug users tend to cycle in and out of settings providing either inpatient or outpatient treatment for addiction, HIV/AIDS, mental health and other comorbidities. While barriers to receive proper HIV care remain for all drug users, active drug users face an even greater synergy of problems to access those life-saving interventions [13, 14].

There is a widely held view that active drug users are poor candidates for HAART because it is believed that drug addiction undermines adherence, or because medical complications and frequent co-infections such as HIV/HCV could make those patients more difficult to treat and less responsive to HAART [19]. It is indeed challenging to provide treatment for HIV-positive active drug users, while patients co-infected with hepatitis $\mathrm{C}$ or tuberculosis present greater clinical needs.
However, those are the ones most in need of comprehensive and timely treatment.

While the World Health Organization recommended that physicians do not discriminate patients on the basis of current or former drug use [20], physicians from Russia and Ukraine, for instance, have declined to treat active drug users HIVpositive and eligible to start HAART [21]. In response to the growing evidence that drug users disproportionately underutilize HIV/AIDS, HCV, addiction prevention and treatment services, recent reviews have urged for equitable and universal access $[14,22]$. Aiming to contribute to this ongoing debate and to evidence-based policies targeting this population, we conducted a systematic review of studies evaluating access to HAART among HIV-positive active drug users.

\section{Methods}

The systematic review was conducted using PRISMA guidelines [23].

\section{Inclusion and Exclusion Criteria}

Studies were included if they were conducted among HIVpositive active drug users or carried out stratified analyses on subsamples of current drug users. Only studies evaluating access to HAART among active DU as a primary outcome and conducted after January 1997 were included. Active drug users were defined as those who have used any illicit drug (except cannabis) over the last six months. Only studies conducting multivariate analysis were selected in order to control for confounding factors, something key in studies dealing with complex psychosocial phenomena, such as addiction and HIV/AIDS treatment access. Studies were excluded if they were based exclusively on qualitative data; were reviews themselves or assessed other populations without disaggregating active drugs users from the overall sample. Studies addressing exclusively alcohol users and/or cannabis smokers were not included.

\section{Data Search}

Search terms that reflect HAART access were identified. Searches combined these terms with Medical Subject Headings (MeSH) for HIV and active drug abuse. MEDLINE via PubMed and Web of Science were searched from 1996 to October 31, 2013. We also reviewed the citation lists of included studies to identify additional eligible references.

\section{Study Selection}

Using a predefined protocol (available from the corresponding author on request), two investigators (M.M., M.R.C.) 
extracted the full text of peer-reviewed papers addressing access to HAART among HIV-positive active DU and assessed their eligibility independently. After all potentially relevant peer-reviewed papers were identified, the two investigators met to achieve consensus.

\section{Data Extraction}

Data extraction was conducted using a standardized form. Data abstractors collected information about the country where the study was conducted, characteristics of the sample (e.g. age, sex, ethnicity), sample size, study design and access to HAART, as well as treatment outcomes such as viral load and CD4 count, when available.

\section{Results}

From the initial searches, 122 peer-reviewed papers were identified. Of these, there was close to perfect agreement between reviewers on the eligibility of 42 papers: 49 did not evaluate HAART access among active drug users, whereas 27 were reviews and four qualitative studies. In a second screening, 21 studies were excluded because of their exclusive use of biological markers as outcomes (i.e. HIV-1 viral load and/or CD4 cell counts). Agreement between reviewers was perfect on the second screening. A third screening excluded seven studies: two presenting repeated data, two conducted before the availability of HAART and three without multivariate analysis. Agreement on the last screening was also perfect. We thus included 14 eligible reports for full data extraction (Fig. 1).

Characteristics of selected studies are summarized in Table 1. In spite of searching for papers published in different languages, all studies identified were published in English and the vast majority were conducted in developed countries (13 of 14). The majority was conducted in North America (six in the United States, five in Canada). One study was conducted in Spain, one in France and one in Ukraine. All selected studies were prospective cohorts and included young adults.

Main results and variables associated with HAART access are presented in Table 2. Each study measured HAART access (or lack of access) using a different strategy, therefore we were unable to present comparisons across studies.

A high percentage of HIV-positive active drug users had never received HIV treatment before the study [24] or received late HIV-diagnosis, therefore initiating HAART at more advanced stages of HIV-infection [25]. Westergaard and colleagues [24] evaluated 790 participants of the AIDS Linked to the Intravenous Experience (ALIVE) study from 1998 through 2011. Authors identified that $93.6 \%$ of study participants were ever linked to care, while $76.7 \%$ had ever received HAART. However, among those only $30.5 \%$ were continuously retained with no 6-month lapses in HIV care and only $10.2 \%$ had sustained viral suppression at every study visit after first receiving HAART. Patients who had lapses in care (gap greater than 6 months without an HIV care visit) were more likely to be active PWID ( $\mathrm{AOR}=1.25$; $95 \% \mathrm{CI}: 1.06-1.49)$ and more likely to be incarcerated in the past 6 months (AOR $=1.49 ; 95 \% \mathrm{CI}: 1.09-2.03)$. When Westergaard and colleagues [24] evaluated healthcare access variables, the strongest predictor of avoiding lapses in HIV-care was having a regular source of primary care (AOR $=0.29 ; 95 \% \mathrm{CI}: 0.17-0.49)$, followed by having the same provider at $>90 \%$ of visits $(\mathrm{AOR}=0.40$; $95 \% \mathrm{CI}: 0.29-0.56)$ and having health insurance (AOR = $0.68 ; 95 \%$ CI:0.52-0.90).

Rodríguez-Arenas et al. [26] evaluated a multicentre hospital-based cohort of HIV-infected patients attending ten hospitals in Spain, including 2621 PWID - 56.4 \% of their participants. PWID had a $33 \%$ lower probability of HAART initiation, when compared to MSM. According to authors fear of less than optimal adherence of active drug users, health professionals' inexperience and misconceptions influence treatment delay are key predictors of late entry into ARV treatment - some physicians delay HAART treatment until the patient was able to 'control his/her addiction'.

A multisite study conducted with 970 HIV-positive participants in the US (71.8\% active drug users), identified that across all types and patterns of drug use, active drug users were more likely to have suboptimal ambulatory care, miss scheduled appointments, use the emergency department, have unmet support services needs, and, as a direct consequence, were less likely to receive HAART once eligible [27].

Inadequate HAART prescription and less than optimal HIV monitoring are also faced by HIV-positive active drug users. Wood and colleagues [28] evaluated a cohort of HIV-positive PWID from British Columbia, a setting providing all medical care free of charge - including HIV-treatment. The authors identified that less than $5 \%$ of HIV-positive active PWID under follow-up had CD4 monitoring consistent with local therapeutic guidelines, while around $20 \%$ received inappropriate antiretroviral prescription.

An innovative study was conducted by Carrieri and colleagues [29]. Researchers evaluated 123 drug users enrolled on the cohort study MANIF, comparing three groups: (1) former PWID, perceived as such (i.e. abstinent patients) by physicians; (2) active PWID not perceived as such by physicians; and (3) active PWID perceived as such (i.e. active drug users) by physicians. According to the study, physicians clearly distinguish PWUDs that they identify as such as "less adherent" patients. However, physicians' perceptions contrast with patients' higher self-reported adherence and lower satisfaction with medical staff - active PWID perceived as such by physicians were the less satisfied. The authors did not actually 
Fig. 1 Flow Diagram of Studies Included in Analysis of HAART Access

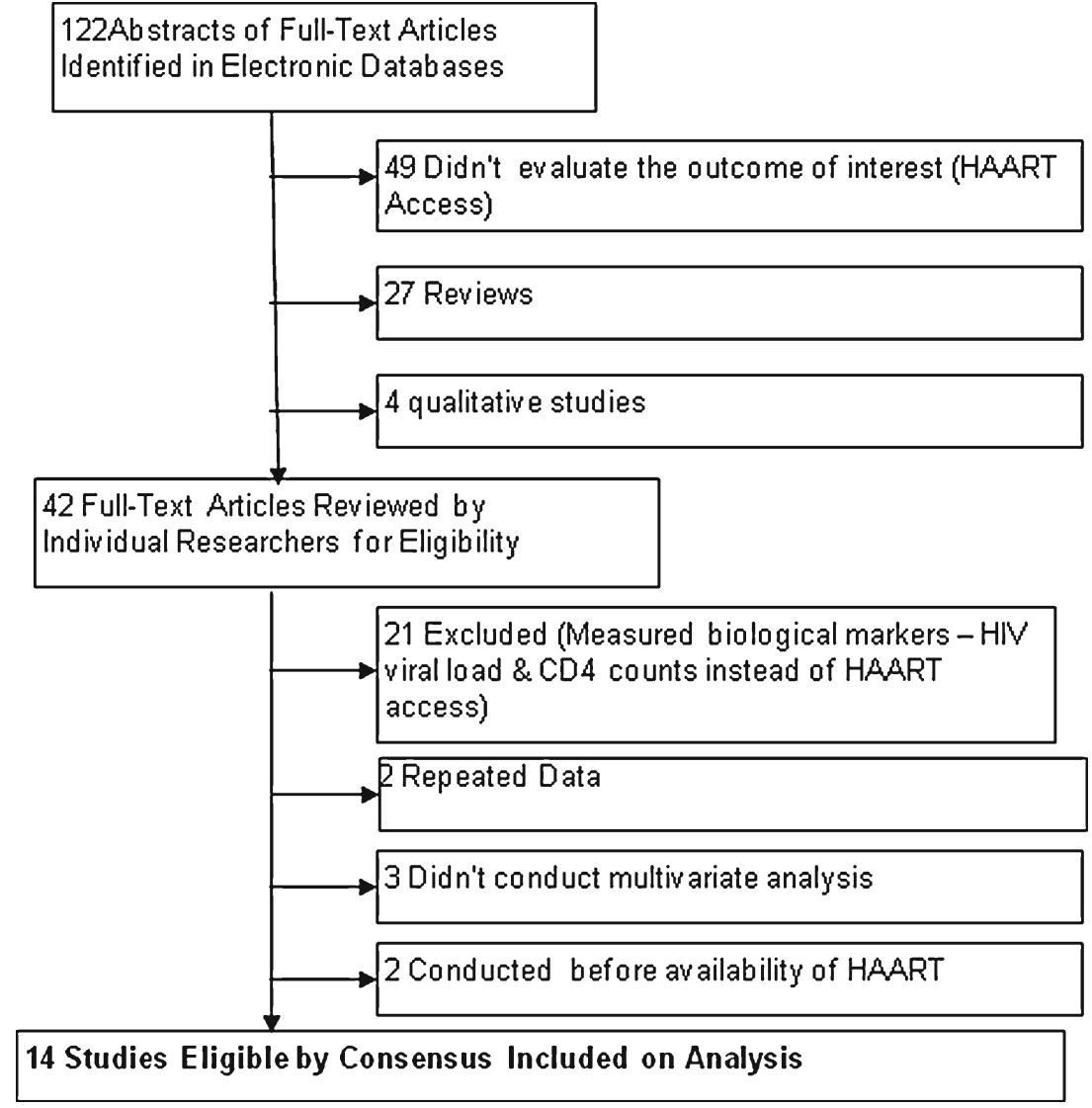

measure stigma and prejudice in the context of patient/ provider relationship. However, those variables could be interpreted as proxy of a differential treatment offered to patients perceived as abstinent, when compared to those perceived as active drug users. Physicians have a major role on HAART access - Strathdee et al. [30] identified that physicians with the least experience were 5.5 times less likely to prescribe HAART for active drug users.

Lack of access to primary care services and frequent experiences of stigma/prejudice within health services, as well as unstable housing and lack of health insurance usually prompt active drug users to rely on emergency department as their first health service option [27,31]. Although factors other than HIV infection also drive emergency department use (e.g. skin and soft tissue infections), the identification of emergency department as the first option for HIV-positive active drug users, usually in need of specialized care, is of great concern in terms of both unreliable provision of care over time and less than optimal use of health services and systems.

According to Fairbairn and colleagues [31], stable living environment facilitate patients ability to stay connected with a primary care service and seek care earlier on disease progression, therefore reducing the use of emergency departments and hospitalizations. The authors suggest that emergency departments should have a better link with specialized services offering infectious disease treatment, mental health and social support strategies.

Strathdee et al. [30] identified a three-fold increased odds for PWID not enrolled in alcohol and/or drug addiction treatment to not receive HAART among those eligible. Arasteh and Des Jarlais [32] also identified increased likelihood of receiving HAART among patients engaged in substance use treatment. An important venue for improving access to HAART is methadone maintenance therapy (MMT), which has been shown to improve access and also adherence to HIVtreatment. Uhlmann et al. [33] conducted a study with 231 antiretroviral-naive HIV-infected opioid-using, identifying that participants on MMT initiated HAART at a significantly elevated rate $(\mathrm{RH}=1.62 ; \mathrm{p}=0.006)$. Tapp et al. [34] identified more than two-fold increased odds of those on MMT to receive and reach $\geq 95 \%$ adherence to HAART $(\mathrm{AOR}=2.35$, $95 \%$ CI: 1.88-2.94, p-value $<0.001)$. MMT has also been associated with more regular monitoring of CD4+ cell counts, an important factor in timing ART initiation [28].

Celentano et al. [25] showed that men enrolled in MMT were $80 \%$ more likely to start HAART, while this effect was not observed among women. More than two-fold increased odds of not receiving HAART among women were also identified by Strathdee et al. [30]. Researchers from the British Columbia Centre for Excellence in HIV/AIDS also 


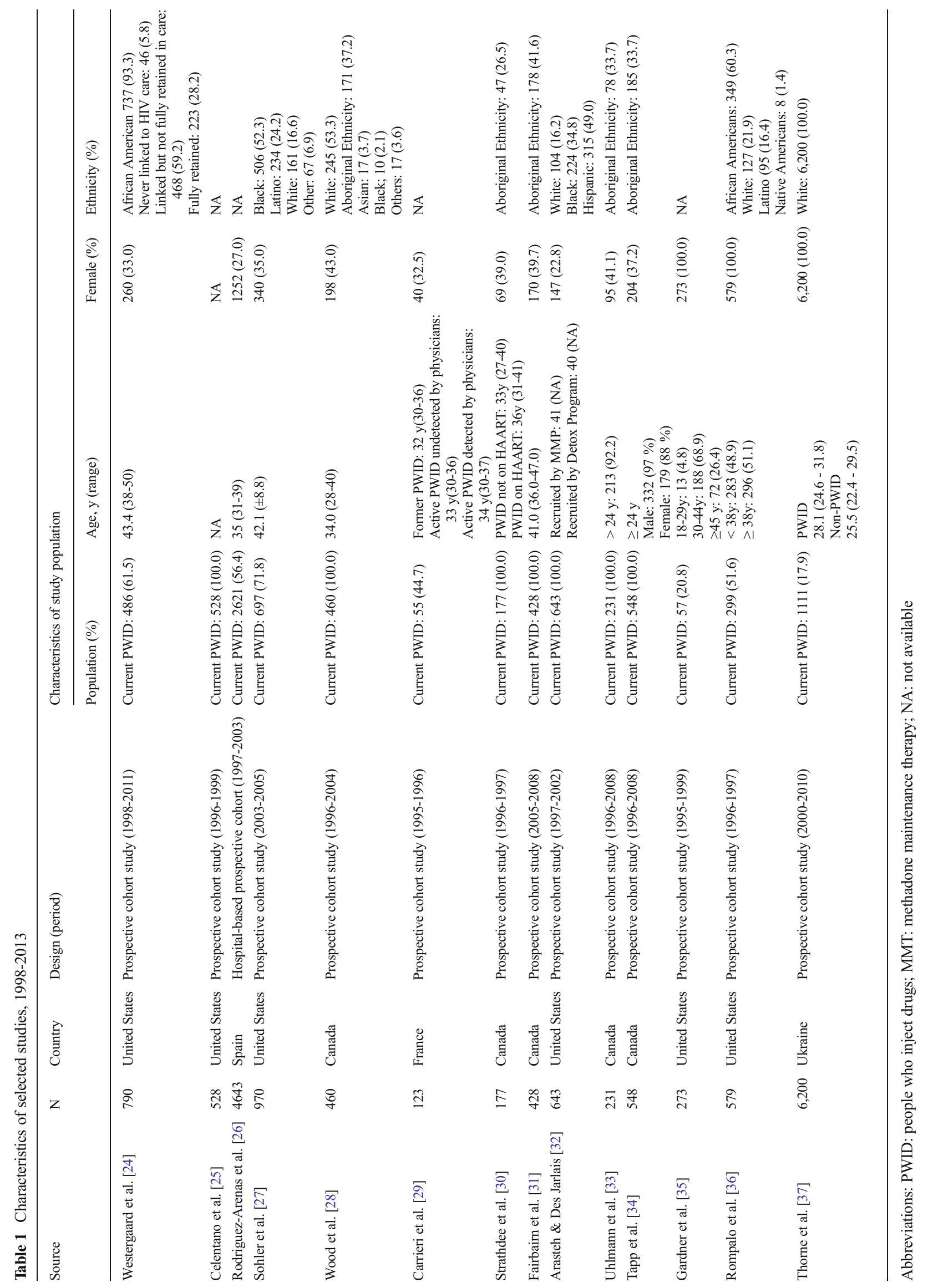




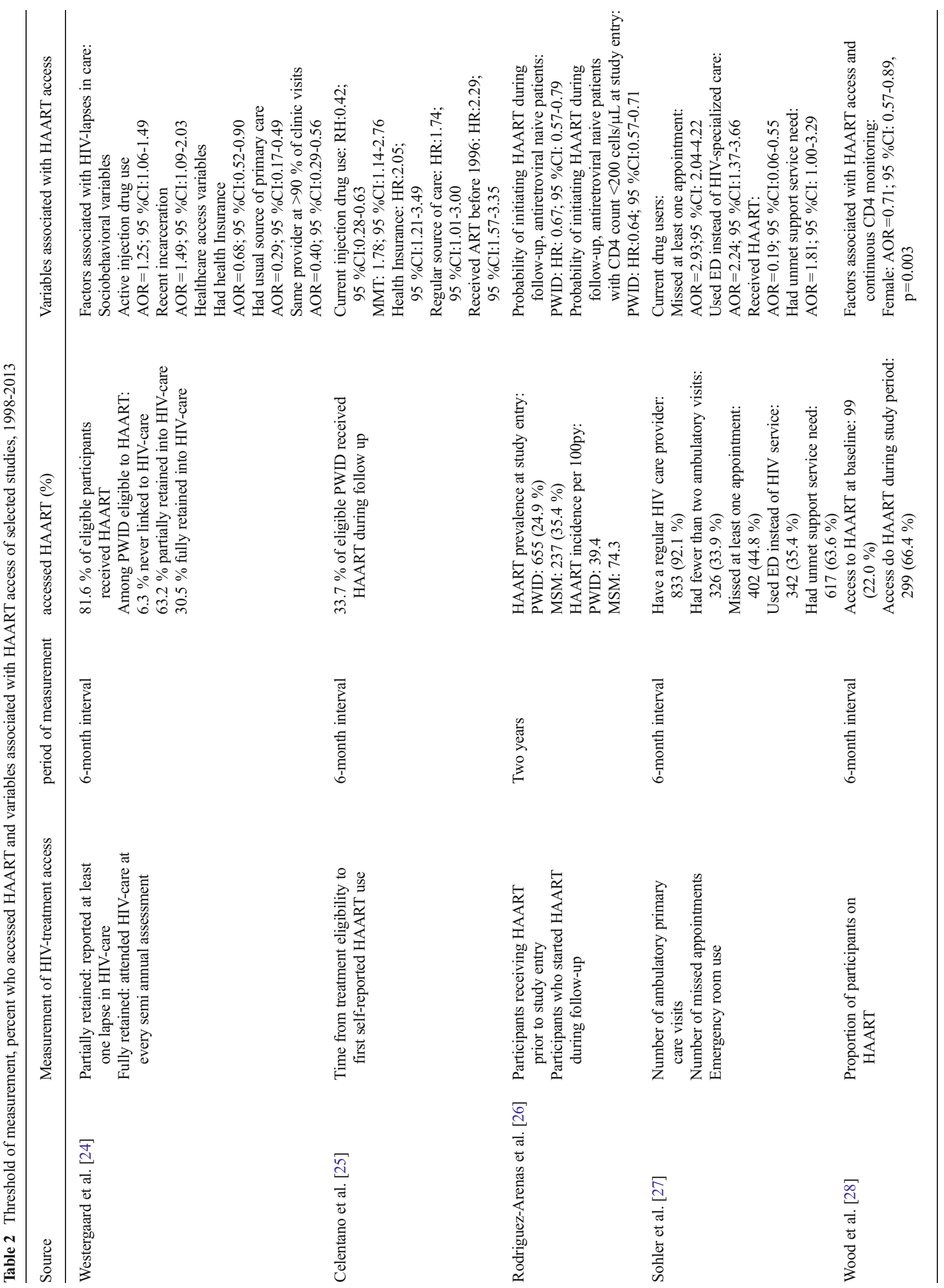




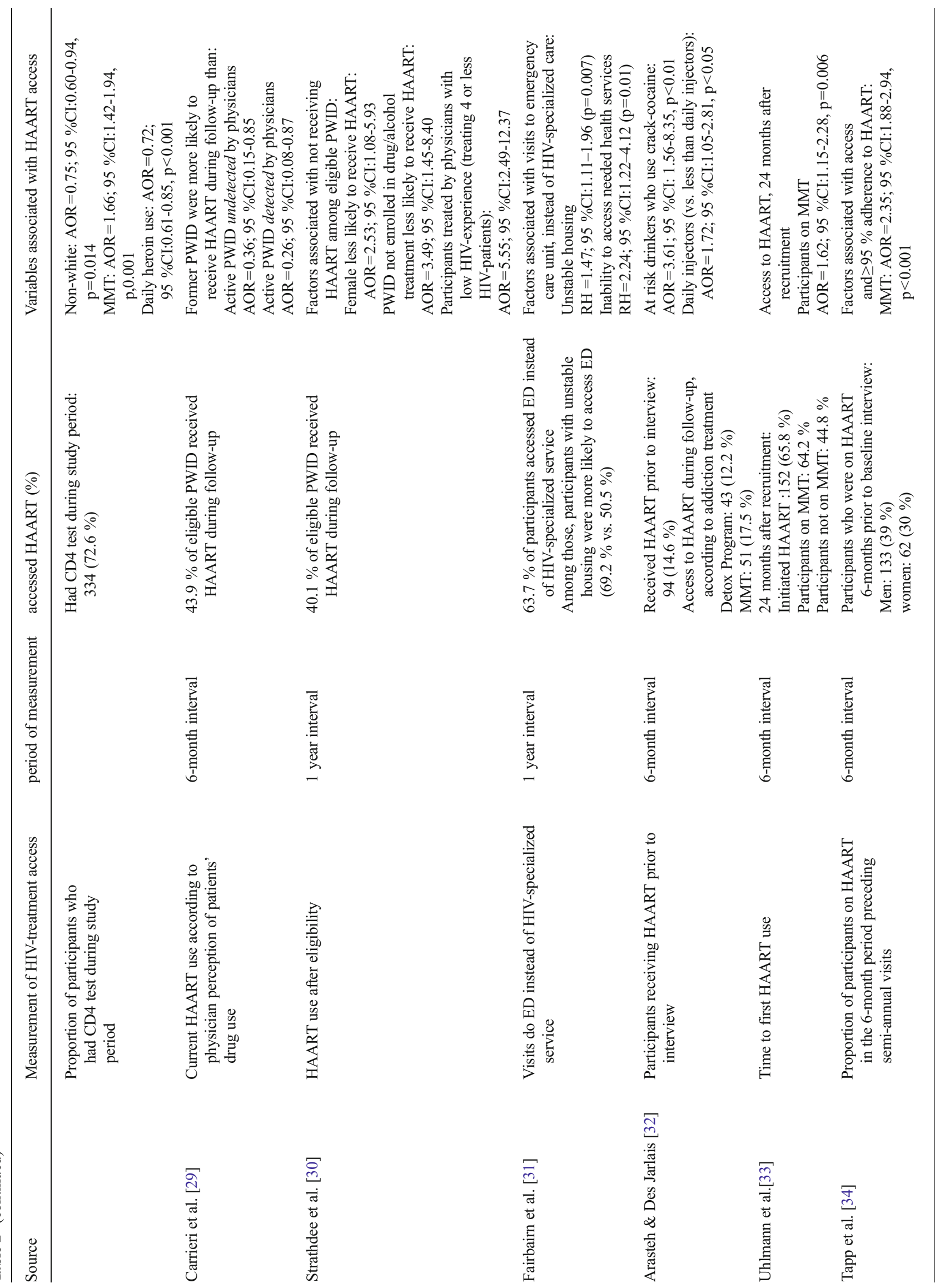




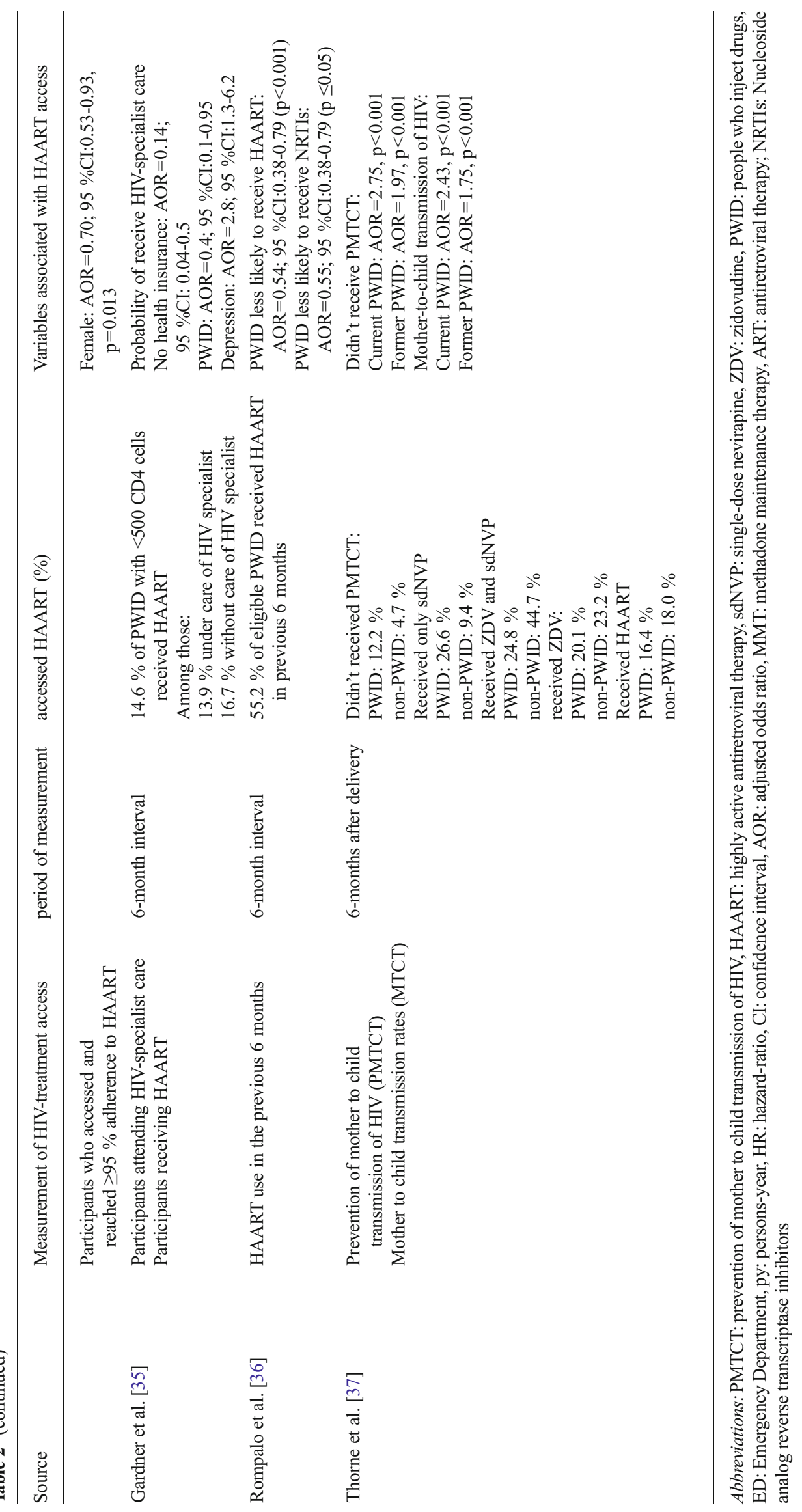


identified lower access to HAART among women [28, 34]. The studies conducted by Gardner et al. [35] and Rompalo et al. [36] showed that women who are currently PWID have fewer indications to start HAART, regardless of using HIV specialist care or not.

A study conducted in Ukraine with 6200 HIV-positive pregnant women [37] identified important challenges faced by female PWID. According to Thorne and colleagues [37], late diagnosis is higher among female PWID: $20 \%$ of new infections were diagnosed intrapartum versus $4 \%$ among nonPWUDs $(\mathrm{P}<0.01)$. PWUDs also had higher rates of preterm delivery and low birth weight: $16 \%$ vs. $7 \% ; 22 \%$ vs. $10 \%$ (P< $0.001])$. PWUDs had nearly three-fold higher odds to receive no neonatal or intrapartum prophylaxis compared with nonPWUDs (OR 2.81, $\mathrm{p}<0.001)$. Mother to child transmission rates were $10.8 \%$ in PWUDs versus $5.9 \%$ in non-PWUDs; and fewer PWUDs with treatment indications received HAART compared with non-PWUDs (58 \% versus $68 \%, \mathrm{P}=0.03$ ).

\section{Discussion}

\section{Conclusion}

Most studies discussed how social and/or structural exposures interact with individual characteristics and behaviours to determine lower access to HAART among HIV-positive active drug users, compared to other populations. According to selected studies, key aspects that might jeopardize this population engagement into HAART are related to the characteristics and operations of health services: limited access to primary care and physicians' stigma/prejudice towards drug users. According to Wolfe [13, pg. 251]: "often IDUs are required to meet the needs of health care systems, and rarely the reverse is true". Strathdee et al. [14] also state that it is urgent to integrate HIV and addiction treatment with prevention strategies such as voluntary counseling and testing and needle exchange programs. Greater and better communication between health care providers and their clientele is pivotal, as well as tailoring medical and psychological procedures and services' characteristics to the special needs of people who misuse drugs/are drug-dependent.

The provision of timely HIV diagnosis and HAART for HIV-positive active drug users is far from impossible, but it calls for a shift in the social framing of patients who are current or former drug users. One should not minimize the challenges of working with active drug users or the multiple needs of those patients, frequently co-infected with hepatitis $\mathrm{C}$ or tuberculosis, usually struggling with psychiatric comorbidities and severe social exclusion. It is necessary to provide proper counseling, treatment and care, while trying to distinguish between the needs of former and current drug users, in order to improve treatment efforts and conduct timely referrals. According to Wolfe [13], greater obstacles to provide adequate treatment and care to HIV-positive drug users are not in individual behaviours and socio-demographic characteristics, but rely mostly in unfriendly health services that usually blame and segregate those most in need of care. According to Strathdee et al. [14, pg. 323]: "Most of all, we need to overcome addictophobia, which manifests as the desire to refer drug users somewhere else, anywhere else, or to deny them access to life-saving interventions 'for their own good.",

HIV-positive active drug users who have been incarcerated, homelessness or with unstable housing were also identified as those facing greater hurdles and difficulties to access HAART. Unstable living conditions are very frequent among active drug users, and might be associated with suboptimal HIV-treatment outcomes. Different contextual dimensions of homelessness, such as food insecurity and lack of space to store medication, as well as the need to prioritize immediate survival over HIVtreatment might also influence HAART initiation and adherence to treatment among this population. Milloy et al. [38] indicate that additional social support interventions (e.g. referral to services providing low-barrier housing alternatives and/or strategies addressing food insecurity) have been shown to increase HAART access, adherence, retention, as well as viral load suppression. A few studies have shown female drug users face additional barriers to engage in HIV-treatment and care, therefore gender-specific interventions should be implemented to address unique barriers such as domestic violence, adequate and timely prenatal care (including comprehensive prevention of mother to child HIV transmission), family planning, fertility desire, among others.

Access to drug addiction treatment was identified as an important facilitator to HAART access in several studies, and MMT contributes to more rapid HAART initiation HAART among opioid-dependent individuals. MMT is a key strategy to address the specific needs of this population, and could be a very effective 'entrance door' for different programs and services. MMT (as well as other low-threshold addiction treatment alternatives) allows regular contact of HIV-positive drug users with health professionals and may allow the health team to offer directly observed therapy for HIV and tuberculosis, among other medical and psychological interventions. Enrollment in MMT or other user-friendly addiction treatment modalities can also mitigate previous negative experiences of stigma and prejudice with health care providers.

This review has a few limitations. We aimed to reduce reviewer bias by conducting abstraction independently, in parallel. However, we did not conduct our review on the socalled 'gray literature' (e.g. non-peer reviewed manuscripts), and therefore publication bias could not be avoided. Qualitative studies were not included in our analysis, but those might bring additional understanding to the complex interplay of drug addiction, HIV, social and contextual factors, and HAART access. Different interpretations of how to measure 
HAART access made between-study comparisons difficult. We identified only one study conducted in developing countries, making it difficult to generalize our findings to those settings. Finally, our review relied upon the information reported in peer-reviewed scientific publications, all published in English. Therefore, these findings are unlikely to represent the treatment experience of a high proportion of HIV-positive PWUDs living in other contexts, such as Russia and eastern Europe, where the HIV epidemic is driven mainly by drugusing populations and access to HAART is uneven $[13,14]$.

HAART has transformed the nature of HIV/AIDS from an imminent death sentence to a chronic, manageable condition. There is also a growing interest in the potential impact of "treatment as prevention" - a notion that expanded coverage with HAART would substantially reduce HIV transmission at the population level. However, late diagnosis and, even more serious, late entry into HAART for eligible patients may compromise and even nullify this putative benefit. Patients who start HAART at more advanced disease stages are more likely to experience HIV-related morbidity and untimely mortality. Optimal timing for HAART initiation is a key strategy for both the individual patient and affected communities. In the absence of a vaccine and cure for HIV, undiagnosed HIV cases and untreated patients represent the biggest challenge in the fight against HIV/AIDS to be fully addressed in the efforts to have an actual AIDS-free generation in the near future.

Degenhardt et al. [39] summarize findings from modeling scenarios about the effect of increased HIV-treatment coverage combined with HIV-prevention interventions targeting PWUDs. The authors showed that when simultaneous scaleup of needle and syringe programs, voluntary counseling and testing, MMT and HAART were implemented, targeting HIVpositive PWID with CD4 cell counts $<350$, HIV incidence could be reduced by up to $63 \%$ within this population. Strathdee and colleagues [14, pg.324] reinforce those findings and state that "providing combination HIV-prevention to IDUs under one umbrella, in a point-of-care, one-stop venue that addresses their myriad needs may seem like a Holy Grail, but it is ultimately what substance users need and is likely to be cost-effective."

More strong political commitments to universal access to HIV-treatment for PWUDs are clearly needed, as well as approaches based on human rights protection which help fight against stigma and discrimination [40]. Future research and interventions targeting PWUDs should also consider broader social and environmental factors that influence lower treatment access, higher morbidity and mortality among HIVpositive PWUDs [41].

\section{Compliance with Ethics Guidelines}

Conflict of Interest Michelle Ralil da Costa declare that she has no conflict of interest.
Francisco Inácio Bastos has received travel/accommodations expenses covered or reimbursed as a reserach for Imperial College, UK (May-July 2013), sponsored by Brazilian agency for postgraduate studies (CAPES). Monica Malta received support from CAPES (grant 0060/ 2013) and CNPq (PAPES VI, grant 407780/2012-2).

Human and Animal Rights and Informed Consent This article does not contain any studies with human or animal subjects performed by any of the authors

\section{References}

1. Quinn TC. HIV epidemiology and the effects of antiviral therapy on long-term consequences. AIDS. 2008;22:S7-S12.

2. Broder $\mathrm{S}$. The development of antiretroviral therapy and its impact on the HIV-1/AIDS pandemic. Antiviral Res. 2010;85:1-18.

3. Achhra AC, Phanuphak P, Amin J. Long-term immunological outcomes in treated HIV-infected individuals in high-income and lowmiddle income countries. Curr Opin HIV AIDS. 2011;6:258-65.

4. Cohen J. Breakthrough of the year: HIV treatment as prevention. Science. 2011;334:1628.

5. CDC. Background brief on the prevention benefits of HIV treatment. http://www.cdc.gov/hiv/pdf/prevention_tap_benefits_of_HIV treatement.pdf Accessed 14 Nov 2013.

6. Montaner JS, Wood E, Kerr T, Lima V, Barrios R, Shannon K, et al. Expanded highly active antiretroviral therapy coverage among HIVpositive drug users to improve individual and public health outcomes. J Acquir Immune Defic Syndr. 2010;55:S5-9.

7. Das M, Chu PL, Santos GM, Scheer S, Vittinghoff E, McFarland W, et al. Decreases in community viral load are accompanied by reductions in new HIV infections in San Francisco. PLoS One. 2010;5:e11068.

8. Miller WC, Hoffman IF, Latkin CA, Strathdee SA, Shoptaw S. HIV antiretroviral prophylaxis for injecting drug users. Lancet. 2013;382: 853.

9. Malta M, Strathdee SA, Magnanini MM, Bastos FI. Adherence to antiretroviral therapy for human immunodeficiency virus/acquired immune deficiency syndrome among drug users: a systematic review. Addiction. 2008;103:1242-57.

10. Friedman SR, West BS, Pouget ER, Hall HI, Cantrell J, Tempalski B, et al. Metropolitan social environments and pre-HAART/HAART era changes in mortality rates (per 10,000 adult residents) among injection drug users living with AIDS. PLoS One. 2013;8:e57201.

11. Wood E, Hogg RS, Yip B, Dong WW, Wynhoven B, Mo T, et al. Rates of antiretroviral resistance among HIV-infected patients with and without a history of injection drug use. AIDS. 2005;19:1189-95.

12. Wood E, Montaner JS, Yip B, Tyndall MW, Schechter MT, O'Shaughnessy MV, et al. Adherence and plasma HIV RNA responses to highly active antiretroviral therapy among HIV-1 infected injection drug users. CMAJ. 2003;169:656-61.

13. Wolfe D. Paradoxes in antiretroviral treatment for injecting drug users: access, adherence and structural barriers in Asia and the former Soviet Union. Int J Drug Policy. 2007;18:246-54.

14. Strathdee SA, Shoptaw S, Dyer TP, Quan VM, Aramrattana A, Substance Use Scientific Committee of the HIV Prevention Trials Network. Towards combination HIV prevention for injection drug users: addressing addictophobia, apathy and inattention. Curr Opin HIV AIDS. 2012;7:320-5.

15. Donoghoe MC, Bollerup AR, Lazarus JV, Nielsen S, Matic S. Access to highly active antiretroviral therapy (HAART) for injecting drug users in the WHO European Region 2002-2004. Int J Drug Policy. 2007; 18:271-80.

16. Grigoryan A, Hall HI, Durant T, Wei X. Late HIV diagnosis and determinants of progression to AIDS or death after HIV diagnosis 
among injection drug users, 33 US States, 1996-2004. PLoS One. 2009;4:e4445.

17. McLellan AT, Lewis DC, O'Brien CP, Kleber HD. Drug dependence, a chronic medical illness: implications for treatment, insurance, and outcomes evaluation. JAMA. 2000;284:1689-95.

18. Arria AM, McLellan AT. Evolution of concept, but not action, in addiction treatment. Subst Use Misuse. 2012;47:1041-8.

19. O'Shaughnessy MV, Hogg RS, Strathdee SA, Montaner JS. Deadly public policy: what the future could hold for the HIV epidemic among injection drug users in Vancouver. Curr HIV/AIDS Rep. 2012;9:394 400 .

20. WHO. HIV/AIDS treatment and care for injecting drug users: Clinical protocol for theWHOEuropean region. Copenhagen: WHO Regional Office for Europe; 2006.

21. Human Rights Watch. Rhetoric and risk: Human rights abuses impeding Ukraine's fight against HIV/AIDS. New York: Human Rights Watch; 2006.

22. Taylor LE, Swan T, Matthews GV. Management of hepatitis C virus/ HIV coinfection among people who use drugs in the era of directacting antiviral-based therapy. Clin Infect Dis. 2013;57:S118-24.

23. Moher D, Liberati A, Tetzlaff J, Altman DG. Preferred reporting items for systematic reviews and meta-analyses: the PRISMA statement. PLoS Med. 2009;6(7):e1000097.

24. Westergaard RP, Hess T, Astemborski J, Mehta SH, Kirk GD. Longitudinal changes in engagement in care and viral suppression for HIV-infected injection drug users. AIDS. 2013;27:2559-66.

25. Celentano DD, Galai N, Sethi AK, Shah NG, Strathdee SA, Vlahov $\mathrm{D}$, et al. Time to initiating highly active antiretroviral therapy among HIV-infected injection drug users. AIDS. 2001;15:1707-15.

26. Rodríguez-Arenas MA, Jarrín I, del Amo J, Iribarren JA, Moreno S, Viciana $\mathrm{P}$, et al. Delay in the initiation of HAART, poorer virological response, and higher mortality among HIV-infected injecting drug users in Spain. AIDS Res Hum Retroviruses. 2006;22:715-23.

27. Sohler NL, Wong MD, Cunningham WE, Cabral H, Drainoni ML, Cunningham CO. Type and pattern of illicit drug use and access to health care services for HIV-infected people. AIDS Patient Care STDS. 2007;21:S68-76.

28. Wood E, Kerr T, Zhang R, Guillemi S, Palepu A, Hogg RS, et al. Poor adherence to HIV monitoring and treatment guidelines for HIVinfected injection drug users. HIV Med. 2008;9(7):503-7.

29. Carrieri MP, Moatti JP, Vlahov D, Obadia Y, Reynaud-Maurupt C, Chesney M. Access to antiretroviral treatment among French HIV infected injection drug users: the influence of continued drug use. MANIF 2000 Study Group. J Epidemiol Community Health. 1999;53:4-8.
30. Strathdee SA, Palepu A, Cornelisse PG, Yip B, O'Shaughnessy MV, Montaner JS, et al. Barriers to use of free antiretroviral therapy in injection drug users. JAMA. 1998;280:547-9.

31. Fairbairn N, Milloy MJ, Zhang R, Lai C, Grafstein E, Kerr T, et al. Emergency department utilization among a cohort of HIV-positive injecting drug users in a Canadian setting. J Emerg Med. 2012;43: 236-43.

32. Arasteh K, Des Jarlais DC. HIV testing and treatment among at-risk drinking injection drug users. J Int Assoc Physicians AIDS Care (Chic). 2009;8:196-201.

33. Uhlmann S, Milloy MJ, Kerr T, Zhang R, Guillemi S, Marsh D, et al. Methadone maintenance therapy promotes initiation of antiretroviral therapy among injection drug users. Addiction. 2010;105:907-13.

34. Tapp C, Milloy MJ, Kerr T, Zhang R, Guillemi S, Hogg RS, et al. Female gender predicts lower access and adherence to antiretroviral therapy in a setting of free healthcare. BMC Infect Dis. 2011;11:86.

35. Gardner LI, Holmberg SD, Moore J, Arnsten JH, Mayer KH, Rompalo A, et al. Use of highly active antiretroviral therapy in HIV-infected women: impact of HIV specialist care. J Acquir Immune Defic Syndr. 2002;29:69-75.

36. Rompalo AM, Shah N, Mayer K, Schuman P, Klein RS, Smith DK, et al. Influence of injection drug use behavior on reported antiretroviral therapy use among women in the HIV Epidemiology Research study: on-site versus referral care. J Acquir Immune Defic Syndr. 2001;28:28-34.

37. Thorne C, Semenenko I, Malyuta R, Ukraine European Collaborative Study Group in EuroCoord. Prevention of mother-to-child transmission of human immunodeficiency virus among pregnant women using injecting drugs in Ukraine, 2000-10. Addiction. 2012;107: 118-28.

38. Milloy MJ, Marshall BD, Kerr T, Buxton J, Rhodes T, Montaner J, et al. Social and structural factors associated with HIV disease progression among illicit drug users: a systematic review. AIDS. 2012;26:1049-63.

39. Degenhardt L, Mathers B, Vickerman P, Rhodes T, Latkin C, Hickman M. Prevention of HIV infection for people who inject drugs: why individual, structural, and combination approaches are needed. Lancet. 2010;376:285-301.

40. Wolfe D, Cohen J. Human rights and HIV prevention, treatment, and care for people who inject drugs: key principles and research needs. J Acquir Immune Defic Syndr. 2010;55 Suppl 1:S56-62.

41. Milloy MJ, Marshall BD, Kerr T, Buxton J, Rhodes T, Montaner J, et al. Social and structural factors associated with HIV disease progression among illicit drug users: a systematic review. AIDS. 2012;26(9):1049-63. 